Wright State University

CORE Scholar

Physics Faculty Publications

Physics

$11-1-1997$

\title{
Spectrum of Hot O At the Exobases of the Terrestrial Planets
}

Jane L. Fox

Wright State University - Main Campus, jane.fox@wright.edu

Aleksander B. Hać

Follow this and additional works at: https://corescholar.libraries.wright.edu/physics

Part of the Physics Commons

\section{Repository Citation}

Fox, J. L., \& Hać, A. B. (1997). Spectrum of Hot O At the Exobases of the Terrestrial Planets. Journal of Geophysical Research-Space Physics, 102 (A11), 24005-24011.

https://corescholar.libraries.wright.edu/physics/15

This Article is brought to you for free and open access by the Physics at CORE Scholar. It has been accepted for inclusion in Physics Faculty Publications by an authorized administrator of CORE Scholar. For more information, please contact library-corescholar@wright.edu. 


\title{
Spectrum of hot $O$ at the exobases of the terrestrial planets
}

\author{
J. L. Fox ${ }^{1}$ \\ Institute for Terrestrial and Planetary Atmospheres, State University of New York at Stony Brook
}

\author{
Aleksander Hać \\ Delphi Chassis Technical Center, Dayton, Ohio
}

\begin{abstract}
The distribution of energetic $\mathrm{O}$ produced in dissociative recombination of $\mathrm{O}_{2}^{+}$at the exobases of the terrestrial planets is important in determining the structure of the outer hot $O$ coronas and for the escape flux of $O$ from Mars. Using recently measured values for the branching ratios of the energetically allowed channels in $\mathrm{O}_{2}^{+}$dissociative recombination, along with models of the vibrational distribution of $\mathrm{O}_{2}^{+}$, we compute the velocity distribution of hot $\mathrm{O}$ atoms produced at the exobases of Mars, Venus, and Earth. We take into account the effects of the ion and electron temperatures, the rotational energy of the ions, and the decrease of the dissociative recombination cross section with relative velocity of the ion and electron. We find that distinct peaks appear at the energies associated with the various channels in the Martian spectrum but that for Venus, the features are less distinct, and for Earth, the distribution appears as a single, but broad and asymmetrical peak. For Mars, we compute the fractions of atoms produced with speeds greater than the escape velocity for ${ }^{16} \mathrm{O},{ }^{17} \mathrm{O}$, and ${ }^{18} \mathrm{O}$ of $0.68,0.64$, and 0.58 , respectively. Thus there is a small isotope effect inherent in the dissociative recombination mechanism that has not been taken into account in models of Martian atmospheric evolution or in estimates of the total inventory of oxygen that is implied by the observed lack of enhancement of the heavier isotopes relative to the terrestrial values.
\end{abstract}

\section{Introduction}

Dissociative recombination (DR) of molecular ions is an important source of hot atoms in the upper atmospheres of the terrestrial planets. In the lower and middle thermospheres, these fast atoms contribute to heating, and near and above the exobases they may travel to great heights, producing hot atom coronas. On Mars, DR of molecular ions may produce atoms with speeds greater than the escape velocity, which is about $4.87 \mathrm{~km} \mathrm{~s}^{-1}$ at the midlatitude exobase.

Rohrbaugh and Nisbet [1973] predicted the existence of a geocorona of energetic $\mathrm{O}$ atoms produced in $\mathrm{DR}$ of $\mathrm{O}_{2}^{+}$and $\mathrm{NO}^{+}$. The corona was detected indirectly by Yee et al. [1980], who measured the intensities and line profiles of the $7320-7330 \AA \mathrm{O}^{+}\left({ }^{2} D\right) \rightarrow \mathrm{O}^{+}\left({ }^{4} S\right)$ emission

\footnotetext{
Dayton, Ohio

Copyright 1997 by the American Geophysical Unıon

Paper number 97JA02089

0148-0227/97/97JA-02089\$09 00
}

${ }^{1}$ Also at Department of Physics, Wright State University, in the twilight airglow. More recently, extended profiles of the atomic oxygen emissions at 1304, 1356, 1027 and $989 \AA$ that were ascribed to hot $O$ were measured from a sounding rocket by Cotton et al. [1993]. The terrestrial hot $O$ corona has been modeled by Yee and Hays [1980], and by Shematovich et al. [1994] and Bisikalo et al. [1995].

The hot oxygen corona of Venus was first observed in extended profiles of $1304 \AA$ dayglow measured by the Pioneer Venus orbiter ultraviolet spectrometer [Nagy et al., 1981]. It has been modeled by Nagy et al. [1981], McElroy et al. [1982], Paxton [1983; see also Paxton and Anderson, 1992], Ip [1988], and Nagy and Cravens [1988]. The hot oxygen corona of Mars has been modeled by Nagy and Cravens [1988], Ip [1988, 1990] and by Lammer and Bauer [1991]. In addition, Zhang et al. [1993] have computed the Martian $O$ coronal density profiles for conditions appropriate to the present and to 2.5 and 3.5 Gyr before present, when the solar flux was more intense by factors of about 3 and 6 , respectively [Zahnle and Walker, 1982]. Reviews of the hot atom coronas of Venus and Mars have been presented 
by Nagy et al. [1990] and by Paxton and Anderson [1992].

Although DR of molecular ions was the first source of energetic $O$ atoms suggested, other sources, including precipitating $\mathrm{O}^{+}$ions [Yee and Hays, 1980; Bisikalo et $a l ., 1995]$ and charge exchange of $\mathrm{O}^{+}$with neutral $\mathrm{O}$ and $\mathrm{H}$ [Nagy et al., 1981], were considered in early models. Recently, Richards et al. [1994] (see also Hickey et al. [1995]) have suggested several additional photochemical sources of hot $\mathrm{O}$ atoms, the most important of which involve quenching and other reactions of the metastable species $\mathrm{O}\left({ }^{1} D\right), \mathrm{O}^{+}\left({ }^{2} D\right)$, and $\mathrm{N}\left({ }^{2} D\right)$. Although many of the proposed reactions are potentially important for populating the inner coronas, $\mathrm{O}_{2}^{+} \mathrm{DR}$ is probably still the most important for populating the outer coronas. It is the only significant source of $O$ atoms with speeds greater than $5 \mathrm{~km} \mathrm{~s}^{-1}$. If they undergo no further collisions, such atoms can attain altitudes greater than 2000 $\mathrm{km}$ above the Earth and Venus, and they can escape from the gravitational field of Mars.

Dissociative recombination of $\mathrm{O}_{2}^{+}$can proceed according to a number of energetically allowed channels, with exothermicities as shown below:

$$
\begin{aligned}
& \mathrm{O}_{2}^{+}+\mathrm{e} \rightarrow \mathrm{O}\left({ }^{3} P\right)+\mathrm{O}\left({ }^{3} P\right)+6.99 \mathrm{eV} \\
& \mathrm{O}_{2}^{+}+\mathrm{e} \rightarrow \mathrm{O}\left({ }^{1} D\right)+\mathrm{O}\left({ }^{3} P\right)+5.02 \mathrm{eV} \\
& \mathrm{O}_{2}^{+}+\mathrm{e} \rightarrow \mathrm{O}\left({ }^{1} S\right)+\mathrm{O}\left({ }^{3} P\right)+2.80 \mathrm{eV} \\
& \mathrm{O}_{2}^{+}+\mathrm{e} \rightarrow \mathrm{O}\left({ }^{1} D\right)+\mathrm{O}\left({ }^{1} D\right)+3.06 \mathrm{eV} \\
& \mathrm{O}_{2}^{+}+\mathrm{e} \rightarrow \mathrm{O}\left({ }^{1} D\right)+\mathrm{O}\left({ }^{1} S\right)+0.835 \mathrm{eV}
\end{aligned}
$$

If the $\mathrm{O}_{2}^{+}$ion is in the ground vibrational and rotational state, each of the $O$ atoms in reactions (1a)-(1e) is released with half the energy shown. The addition of one vibrational quantum adds about $0.23 \mathrm{eV}$ to the exothermicity. There are slight differences in the vibrational and rotational energies of ${ }^{18} \mathrm{O}^{16} \mathrm{O}^{+},{ }^{17} \mathrm{O}^{16} \mathrm{O}^{+}$, and ${ }^{32} \mathrm{O}_{2}^{+}$, that lead to small differences in the exothermicities presented above for the heavier isotopes. In some models, it has been assumed that all the DR's proceeded via channel (1b) [e.g., McElroy et al., 1982; $I p, 1988]$, an approximation that was roughly consistent (but not uniquely so) with the yields measured by Zipf [1970] of 0.1 and 0.9 for $O\left({ }^{1} S\right)$ and $O\left({ }^{1} D\right)$, respectively. Zipf [1988] later withdrew these values because the vibrational distribution of $\mathrm{O}_{2}^{+}$in the experiment was unknown. Rohrbaugh and Nisbet [1973] derived quite different branching ratios from the measurements of Zipf [1970] for their model of the O corona. Paxton [1983] determined branching ratios by fitting a model of the Venus hot $O$ corona to Pioneer Venus measurements of the $1304 \AA$ dayglow intensity profiles.

Guberman $[1987,1988]$ carried out large ab initio calculations of the cross sections and rate coefficients for channels (1b)-(1e). He showed that the yield for channel (1c) should be negligible for low vibrational levels of $\mathrm{O}_{2}^{+}$. Because he did not compute cross sections for channel (1a), which leads to two ground state atoms, branching ratios could not be derived from his calculations alone. Theoretically, however, the branching ratio for channel (1a) could be determined as the difference between the measured total rate coefficient [Alge et al., 1983; Mul and McGowan, 1979] and the rate coefficients for channels (1b)-(1e) from Guberman [1987, 1988]. Fox [1990] (see also Fox and Bougher [1991]) applied this method to derive yields appropriate to the temperatures of the Venus exobase. In practice, however, since the vibrational distribution of the $\mathrm{O}_{2}^{+}$ions in the experiment is unknown, the measured total rate coefficient may not be applicable to the model conditions. In fact, this procedure was found to lead to negative values for the yield of channel (1a) at high electron temperatures [Fox, 1993].

Recently, Kella et al. [1997] have measured the branching ratios for channels (1a)-(1e) in a heavy-ion storage ring for $\mathrm{O}_{2}^{+}(v=0)$ and for $\mathrm{O}_{2}^{+}$in an extended vibrational distribution. Their results are compared to

\begin{tabular}{|c|c|c|c|c|c|c|c|c|}
\hline \multirow[b]{2}{*}{ Channel } & \multirow{2}{*}{$\begin{array}{l}\text { Energy, } \\
\mathrm{eV}\end{array}$} & \multicolumn{5}{|c|}{ Yield } & \multirow[b]{2}{*}{ f } & \multirow[b]{2}{*}{ g } \\
\hline & & $\mathbf{a}$ & $\mathbf{b}$ & c & d & e & & \\
\hline${ }^{3} P+{ }^{3} P$ & 6.99 & 0.325 & 0.0 & 0.15 & 0.22 & 0.334 & $0.22 \pm 0.10$ & 0.25 \\
\hline${ }^{3} P+{ }^{1} D$ & 5.02 & 0.30 & 1.0 & 0.85 & 0.55 & 0.393 & $0.42 \pm 0.05$ & 0.39 \\
\hline${ }^{3} P+{ }^{1} S$ & 2.80 & 0.05 & 0.0 & 0.0 & 0.0 & 0.0 & $<0.01$ & - \\
\hline${ }^{1} D+{ }^{1} D$ & 3.05 & 0.275 & 0.0 & 0.0 & 0.13 & 0.233 & $0.31 \pm 0.07$ & 0.27 \\
\hline${ }^{1} D+{ }^{1} S$ & 0.835 & 0.05 & 0.0 & 0.0 & 0.10 & 0.038 & $0.05 \pm 0.02$ & 0.09 \\
\hline
\end{tabular}
those derived in other ways in Table 1. It can be seen that the branching ratios derived by Rohrbaugh and

Table 1. Branching Ratios for Various Channels in Dissociative Recombination of $\mathrm{O}_{2}^{+}$Computed or Assumed in Calculations of the Hot Oxygen Coronas, Compared With the Measured Values

Yields a: derived by Rohrbaugh and Nisbet [1973] from data of Zipf [1970]. Used by Nagy et al. [1981] and Nagy and Cravens [1988]. Yields b: assumed by McElroy [1972], and McElroy et al. [1982], probably as a rough fit to measured yields of Zipf [1970]. Also adopted by $I p[1988,1990]$. Yields c: assumed by Shematovich et al. [1994] and Bisikalo et al. [1995]. Yields d: determined by Paxton [1983; see also Paxton and Anderson, 1992] from a best fit to the PV OUVS $1304 \AA$ profiles. Adopted by Nagy and Cravens [1988] and by Zhang et al. [1993]. Yields e: derived by $F_{0 x}$ [1990] (see also, Fox and Bougher [1991]) from the calculations of Guberman [1987, 1988] and the measurements of $M u l$ and McGowan [1979] for the Venus exobase. Used by Hodges et al. [1993]. Yields f: measured by Kella et al. [1997] for $\mathrm{O}_{2}^{+}$in the vibrational ground state. Yields g: measured by Kella et al. [1997] for a vibrationally extended distribution. 
Nisbet [1973] and by Fox [1990] are consistent with the measured values to within the error bars of the experiment. The branching ratio derived by Paxton [1983] for channel (1a), 0.22, is in excellent agreement with the measurements, probably because that channel alone determines the morphology of the outer reaches of the corona. Because of the multiplicity of sources, however, the coronal morphology is less sensitive to the branching ratios for the lower exothermicity channels. It is also interesting to note that the yields for $O\left({ }^{3} P\right), O\left({ }^{1} D\right)$, and $O\left({ }^{1} S\right)$, of $0.89,1.02$, and 0.09 , respectively, derived from the Kella et al. [1997] measurements for $\mathrm{O}_{2}^{+}$in an extended vibrational distribution are remarkably similar to those reported by Zipf [1970].

We compute here the spectrum of $O$ atoms released in dissociative recombination of $\mathrm{O}_{2}^{+}$at the exobases of Mars, Venus, and Earth by combining our models of the vibrational distribution of $\mathrm{O}_{2}^{+}$with branching ratios for channels (1a)-(1e) taken from the recent measurements of Kella et al. [1997]. We also take into account the variation of cross section for the $\mathrm{DR}$ of $\mathrm{O}_{2}^{+}$with relative velocity of the ion and electron as reported by Walls and Dunn [1974], the rotational distribution of the $\mathrm{O}_{2}^{+}$ions, and the spread of velocities of the ion and electron. The latter effects are incorporated by carrying out Monte Carlo calculations in which the rotational energy of the $\mathrm{O}_{2}^{+}$and the initial velocities of the ion and electron are chosen from among a distribution characteristic of the ion and electron temperatures appropriate to the exobase. For Mars, we derive the fraction of dissociative recombinations that lead to escape for ${ }^{16} \mathrm{O},{ }^{17} \mathrm{O}$, and ${ }^{18} \mathrm{O}$ and the resulting isotope fractionation factors.

\section{Calculations}

In order to determine the exothermicity of a DR reaction, it is necessary to know the vibrational and rotational energy of the ion. We have modeled the vibrational distribution of $\mathrm{O}_{2}^{+}$in the thermospheres of Venus, Earth, and Mars in a similar manner to that described by Fox $[1985,1986]$. A brief summary is presented here. Near the terrestrial exobase, which is at about $450-500$ $\mathrm{km}$, vibrationally excited $\mathrm{O}_{2}^{+}$is produced mainly in the reactions

$$
\begin{gathered}
\mathrm{O}^{+}+\mathrm{O}_{2} \rightarrow \mathrm{O}_{2}^{+}+\mathrm{O}+1.55 \mathrm{eV} \\
\mathrm{N}^{+}+\mathrm{O}_{2} \rightarrow \mathrm{O}_{2}^{+}+\mathrm{N}\left({ }^{4} S\right)+2.46 \mathrm{eV}
\end{gathered}
$$

Where no other information is available, the energetically accessible vibrational levels are assumed to be populated equally. Direct production by photoionization and photoelectron impact ionization of $\mathrm{O}_{2}$ is less important at these altitudes.

At the exobases on Mars and Venus, which are near $200 \mathrm{~km}$, the most important sources of vibrationally excited $\mathrm{O}_{2}^{+}$are the reactions

$$
\mathrm{O}^{+}+\mathrm{CO}_{2} \rightarrow \mathrm{O}_{2}^{+}+\mathrm{CO}+1.17 \mathrm{eV}
$$

$$
\mathrm{CO}_{2}^{+}+\mathrm{O} \rightarrow \mathrm{O}_{2}^{+}+\mathrm{CO}+1.35 \mathrm{eV} \text {. }
$$

The vibrational distribution produced in reaction (4) has been reported by Hunton et al. [1991] and by Walter et al. [1993]. Here we have adopted the nascent distribution reported by Hunton et al. [1991]. In all three ionospheres, vibrational levels are interchanged in quenching and collisional excitation by neutral species. The most important quencher near the exobases is $O$, and we have assumed that the reaction

$$
\mathrm{O}+\mathrm{O}_{2}^{+}(v) \rightarrow \mathrm{O}_{2}^{+}(v-1)+\mathrm{O}
$$

proceeds with a rate coefficient of $1 \times 10^{-10} \mathrm{~cm}^{3} \mathrm{~s}^{-1}$. Fluorescent scattering of radiation in the $\mathrm{O}_{2}^{+}\left(A^{2} \Pi_{u} \rightarrow\right.$ $X^{2} \Pi_{g}$ ) second negative band system is included in the model but is found to be important only for vibrational levels $v>\mathbf{7}$ for Earth and $v>5$ for Venus and Mars.

The computed vibrational distributions are shown in Table 2. The distribution is slightly more extended at the terrestrial exobase than at the Venus exobase. The higher exospheric temperatures in the terrestrial thermosphere lead to exobase neutral densities that are smaller by a factor of $\sim 2$ than those for Venus or Mars. Some of the differences in detail also result from the production of $\mathrm{O}_{2}^{+}$by direct ionization of $\mathrm{O}_{2}$, which is significant only for the Earth. Also, the highest vibrational level that can be produced in reaction (2) is $v=7$, whereas the highest for reaction (4) is $v=5$, and this results in the relatively higher probabilities for $v=6$ and $v=7$ for the terrestrial case.

The computed vibrational distribution at the Martian exobase is more relaxed than that of either the Earth or Venus. Although the total neutral density is about the same at the exobases of Mars and Venus, and thus the quenching rates are similar, the $\mathrm{O}_{2}^{+}$lifetime against DR on Mars is longer, about $1000 \mathrm{~s}$, as compared to $60 \mathrm{~s}$ for Earth and $100 \mathrm{~s}$ for Venus. Thus an $\mathrm{O}_{2}^{+}$ion at the Martian exobase, on average, undergoes a larger number of quenching collisions during its lifetime than it would at the terrestrial or Venus exobases.

Monte Carlo calculations were carried out to determine the distribution of velocities of the product $O$ atoms for $4 \times 10^{4}$ events. A more complete description of the Monte Carlo calculations can be found in the work

Table 2. Computed Vibrational Distribution of $\mathrm{O}_{2}^{+}$for $v \leq 8$ at the Exobases of the Terrestrial Planets

\begin{tabular}{llll}
\hline & \multicolumn{3}{c}{ Fraction } \\
\cline { 2 - 4 }$v$ & Venus & Earth & Mars \\
\hline 0 & 0.43 & 0.34 & 0.80 \\
1 & 0.26 & 0.18 & 0.074 \\
2 & 0.12 & 0.16 & 0.043 \\
3 & 0.077 & 0.066 & 0.035 \\
4 & 0.061 & 0.060 & 0.025 \\
5 & 0.039 & 0.058 & 0.015 \\
6 & 0.0036 & 0.054 & 0.0047 \\
7 & 0.00092 & 0.047 & 0.00027 \\
8 & 0.00070 & 0.0042 & 0.00021 \\
\hline
\end{tabular}


of Fox and Hać [1997], where the analogous calculation for $\mathrm{DR}$ of $\mathrm{N}_{2}^{+}$is described. A brief summary is presented here. The initial speeds of the ion and electron in the laboratory frame were determined by generating random numbers from a Gaussian distribution for the $x, y$, and $z$ components of the velocities. Although they could have been derived from this information, the polar and azimuthal angles were determined separately by generating two more random numbers. This choice was arbitrary, and the resulting angular distribution would have been the same using either method. The initial velocities of the ion and electron were then decomposed into the velocity of the center of mass and the velocities in the center-of-mass system. The angular distribution of the final velocities of the product $O$ atoms in the center-of-mass system was assumed to be isotropic and was determined by generating random numbers for the polar and azimuthal angles. It should be noted that the assumption of isotropy is a source of uncertainty in the model, but little information is available about the angular distribution of products of DR. Once the directions of the final velocities are specified, the magnitudes of the velocities are determined by conservation of energy and momentum.

The effect of the rotational energy of the $\mathrm{O}_{2}^{+}\left({ }^{2} \Pi_{1 / 2,3 / 2}\right)$ ions was also included, with rotational constants taken from Laher and Gilmore [1991]. Rotational quantum numbers $J$ for $\mathrm{O}_{2}^{+}\left({ }^{2} \Pi_{1 / 2}\right)$ from $1 / 2$ to $81 / 2$ and for $\mathrm{O}_{2}^{+}\left({ }^{2} \Pi_{3 / 2}\right)$ from $3 / 2$ to $81 / 2$ were included. The range 0 to 1 was divided into intervals (labeled by $J$ ) in which the size of each interval was proportional to the probability for a given $J$ value. A random number was generated and the $J$ value was determined by the interval into which the number fell.

The cross section for DR decreases with the relative velocity $v$ of the ion and electron, which causes lower energy collisions to be slightly more probable. We adopted the cross section $\sigma(v)$ from Walls and Dunn [1974], who reported that for relative velocities less than about 600 $\mathrm{km} \mathrm{s}^{-1}, \sigma(v) \propto v^{-2.3 \pm 0.3}$. The probability of DR occurring is proportional to $v \sigma(v)$. Although the experimental cross sections apply strictly only to the vibrational distribution in the ion storage trap, the probabilities were assumed to apply to all vibrational levels. A random number chosen from a uniform distribution on the interval 0 to 1 was generated and compared to a suitably normalized probability for that value of $v$. The event was counted only if the random number was less than the normalized probability. For example, if the relative velocity of the ion and electron is $100 \mathrm{~km} \mathrm{~s}^{-1}$, the normalized probability is 0.5179 , and the event is counted if the random number is less than 0.5179 but is discarded if it is larger. It should be noted that the absolute value of this probability is meaningless; only relative values matter.

The branching ratios for individual excited vibrational levels of $\mathrm{O}_{2}^{+}$were not measured by Kella et al.
[1997], and values were reported only for the vibrational ground state and for an extended vibrational distribution, which was not expected to be equilibrated with the kinetic or rotational temperature (L. H. Anderson, private communication, 1997). In order to estimate the branching ratios for the excited vibrational levels, we have somewhat arbitrarily assumed that half the ions were excited in their experiment, and that the branching ratios for all the vibrational levels with $v>0$ are the same. These assumptions lead to yields of $0.28,0.36$, 0.23 , and 0.13 for channels (1a), (1b), (1d), and (1e), respectively, for $\mathrm{O}_{2}^{+}(v>0)$. In fact, however, the fraction of ions in vibrationally excited states in the storage ring may be larger than one half. If the ions are produced by electron impact ionization in a Franck-Condon distribution, the the fraction of excited ions in the ground state could be as low as $\mathbf{1 8 \%}$.

The ion temperature $\left(T_{i}\right)$ and electron temperature $\left(T_{e}\right)$ were assumed to be 400 and $2000 \mathrm{~K}$, respectively, at the Martian exobase, which is near $200 \mathrm{~km}$ for Viking conditions of low solar activity [Hanson et al., 1977; Hanson and Mantas, 1988]. Pioneer Venus in situ measurements were at high solar activity; $T_{i}=500 \mathrm{~K}$ and $T_{e}=3000 \mathrm{~K}$ near the $210 \mathrm{~km}$ dayside exobase [e.g., Miller et al., 1980]. In the altitude range 450-500 km, where the terrestrial exobase is located, $T_{i}$ and $T_{e}$ are about 1200 and $2700 \mathrm{~K}$, respectively [e.g., Bilitza and Hoegy, 1990]. The rotational temperature $T_{r}$ of the of the $\mathrm{O}_{2}^{+}$ions was assumed to be the same as $T_{i}$. Although the rotational populations may not be equilibrated with the kinetic temperatures, we found that the rotational energy does not have a significant effect on the $O$ distribution for $T_{r}=T_{i}$, and the results were insensitive to the assumed rotational temperature for variations about that value of a factor of 2 or more.

\section{Results and Discussion}

The velocity distributions for product $\mathrm{O}$ atoms in $\mathrm{O}_{2}^{+}$ DR are presented in Figures 1, 2, and 3 for Mars, Venus and Earth, respectively. Above each velocity distribution we show the maximum altitude that an $\mathrm{O}$ atom released vertically in dissociative recombination of $\mathrm{O}_{2}^{+}$can attain. For Mars, the velocity distributions are shown for ${ }^{18} \mathrm{O},{ }^{17} \mathrm{O}$, and ${ }^{16} \mathrm{O}$ and the vertical line in Figure 1 is the escape velocity at midlatitudes, $4.87 \mathrm{~km} \mathrm{~s}^{-1}$. Individual peaks arising from the channels (1a), (1b), (1d), and (1e) are clearly visible, although they are broadened considerably. Most of the broadening is due to the spread of velocities of the ion, although the presence of vibrational excitation of the $\mathrm{O}_{2}^{+}$is also important. The fraction of $O$ atoms with velocities exceeding the escape velocity are $0.68,0.64$, and 0.58 for ${ }^{16} \mathrm{O},{ }^{17} \mathrm{O}$, and ${ }^{18} \mathrm{O}$, respectively. Thus there is a small isotope effect inherent in the DR mechanism, which operates in addition to the effect of depletion of the heavier isotopes at the exobase that results from diffusive separation above 

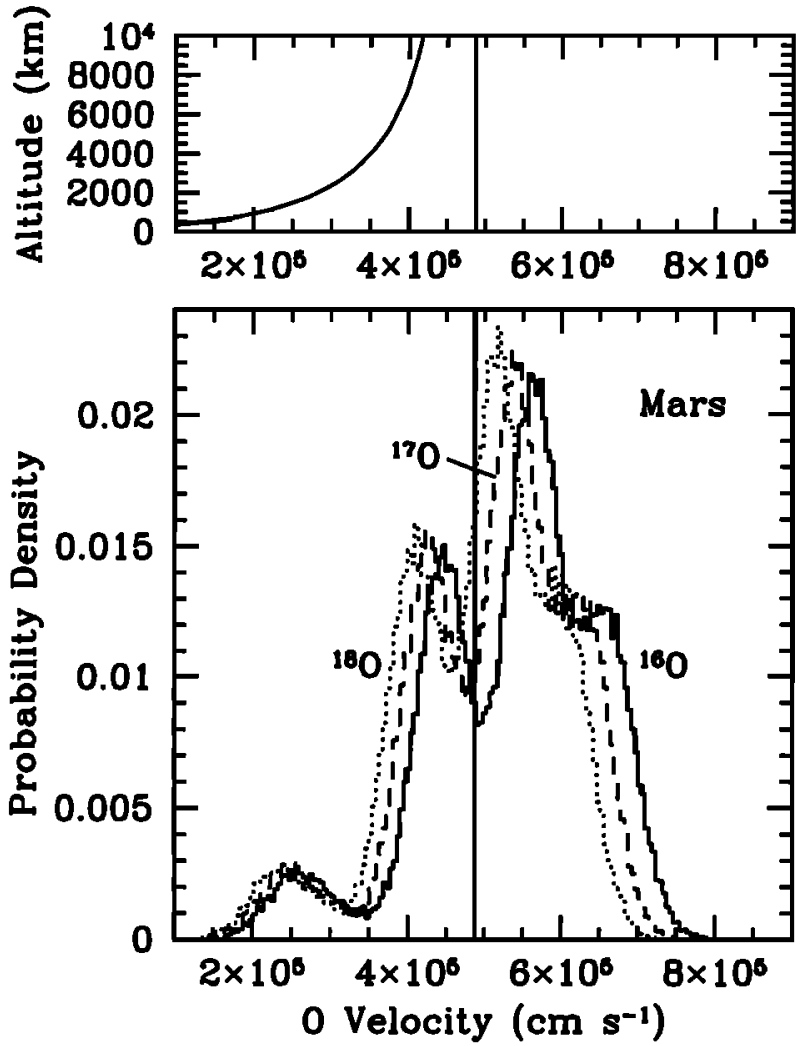

Figure 1. (top) Maximum altitude to which an $O$ atom released vertically at the Martian exobase can rise as a function of velocity. The vertical line indicates the escape velocity at the midlatitude exobase. (bottom) Nascent velocity distribution of ${ }^{16} \mathrm{O},{ }^{17} \mathrm{O}$, and ${ }^{18} \mathrm{O}$ atoms produced in DR of $\mathrm{O}_{2}^{+}$at the Martian exobase, near $200 \mathrm{~km}$. The ion temperature is $400 \mathrm{~K}$, and the electron temperature is $2000 \mathrm{~K}$.

the homopause. The fractionation factor $R$, which is the ratio of the escape probability of a heavy isotope to that of ${ }^{16} \mathrm{O}$, is thus 0.93 for ${ }^{17} \mathrm{O}$ and 0.84 for ${ }^{18} \mathrm{O}$. This effect has not been taken into account in models [McElroy and Yung, 1976; Jakosky, 1991]. In particular, the inferred total oxygen inventory that is required by the measured lack of enhancement of heavier oxygen isotopes in the Martian atmosphere relative to the terrestrial values [Nier et al, 1976] will be larger than previous models have assumed.

We can compare our escape probabilites to those computed analytically by Wallis [1978]. He found that essentially all $O$ atoms produced in channel (1a) had sufficient energy to escape, and for channel (1b), the fractions with velocities greater than the escape velocities were 1.0 for ${ }^{16} \mathrm{O}$ and 0.995 for ${ }^{18} \mathrm{O}$. Our calculations confirm the lack of an isotope effect for channel (1a), but for channel (1b), our computed escape fractions for ${ }^{16} \mathrm{O}$ and ${ }^{18} \mathrm{O}$ are 0.991 and $\mathbf{0 . 8 3 3}$, respectively, leading to a fractionation factor of 0.84 . The reason for the disagreement is uncertain, but a similar difference was found between our computed escape fractions for ${ }^{15} \mathrm{~N}$ and ${ }^{14} \mathrm{~N}$ in $\mathrm{N}_{2}^{+} \mathrm{DR}$ [Fox and Hać, 1997] and those of Wallis [1978].

The spectrum for ${ }^{16} \mathrm{O}$ and ${ }^{18} \mathrm{O}$ at the Venus exobase is shown in Figure 2 and is similar to the Mars spectrum, except that the peaks for the different channels are not as distinct. This is mostly due to the higher ion temperature, but partly also to the more extended vibrational distribution. The average ${ }^{16} \mathrm{O}$ velocity is $5.495 \mathrm{~km} \mathrm{~s}^{-1}$. Also shown as a dashed curve here is the velocity distribution for the case with no variation of the probability of dissociative recombination with relative velocity of the ion and electron. The velocities for ${ }^{16} \mathrm{O}$ are only slightly larger for case, averaging $\mathbf{5 . 5 7 6}$ $\mathrm{km} \mathrm{s}^{-1}$. Thus there is little sensitivity to the exact form of the assumed variation of the DR cross section with relative velocity.

The terrestrial spectrum, shown in Figure 3, exhibits only a single, albeit broad and quite asymmetrical peak that maximizes at about $6 \times 10^{5} \mathrm{~cm} \mathrm{~s}^{-1}$. The peak for channel (1a) is barely visible as a shoulder on the high-

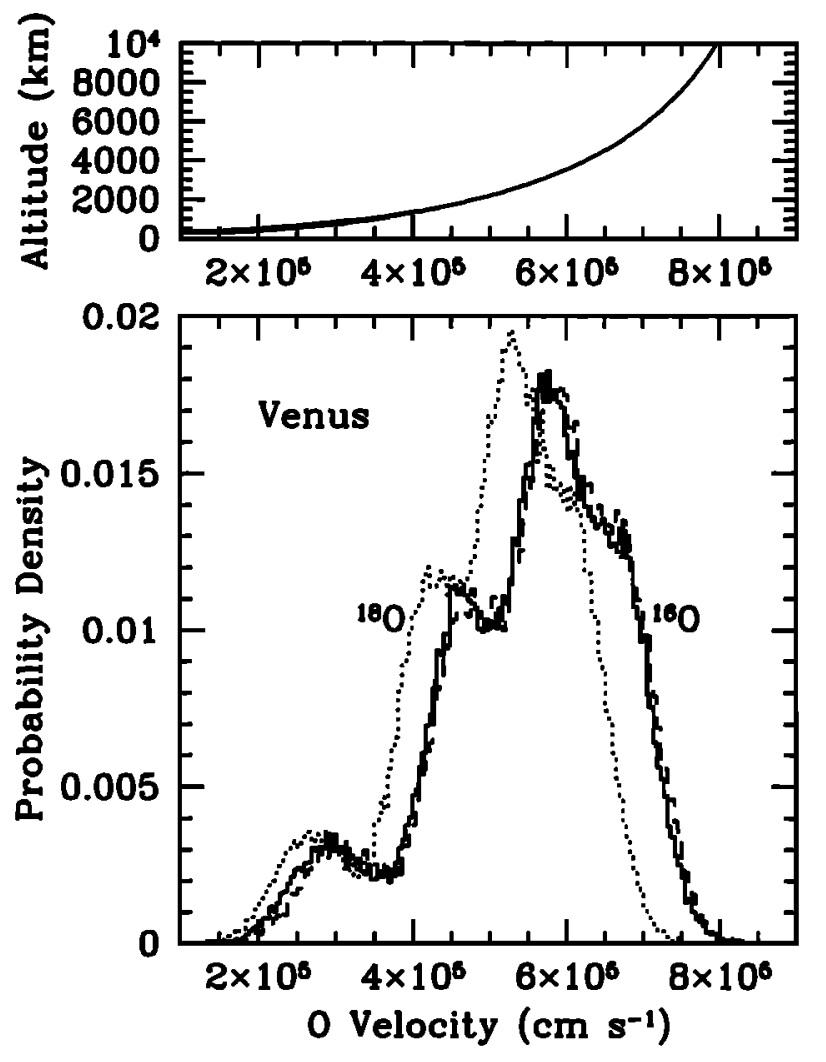

Figure 2. (top) Maximum altitude to which an $O$ atom released vertically at the Venus exobase can rise as a function of velocity. (Bottom) Nascent velocity distribution of ${ }^{18} \mathrm{O}$ and ${ }^{16} \mathrm{O}$ at the Venus exobase, near $210 \mathrm{~km}$, for $T_{i}=500 \mathrm{~K}$, and $T_{e}=3000 \mathrm{~K}$. The peaks for the channels (1a)-(1e) are considerably broadened over the Mars values. The dashed curve shows the velocity distribution for the case in which it is assumed that there is no variation of the probability of dissociative recombination with relative velocity of the ion and electron. 


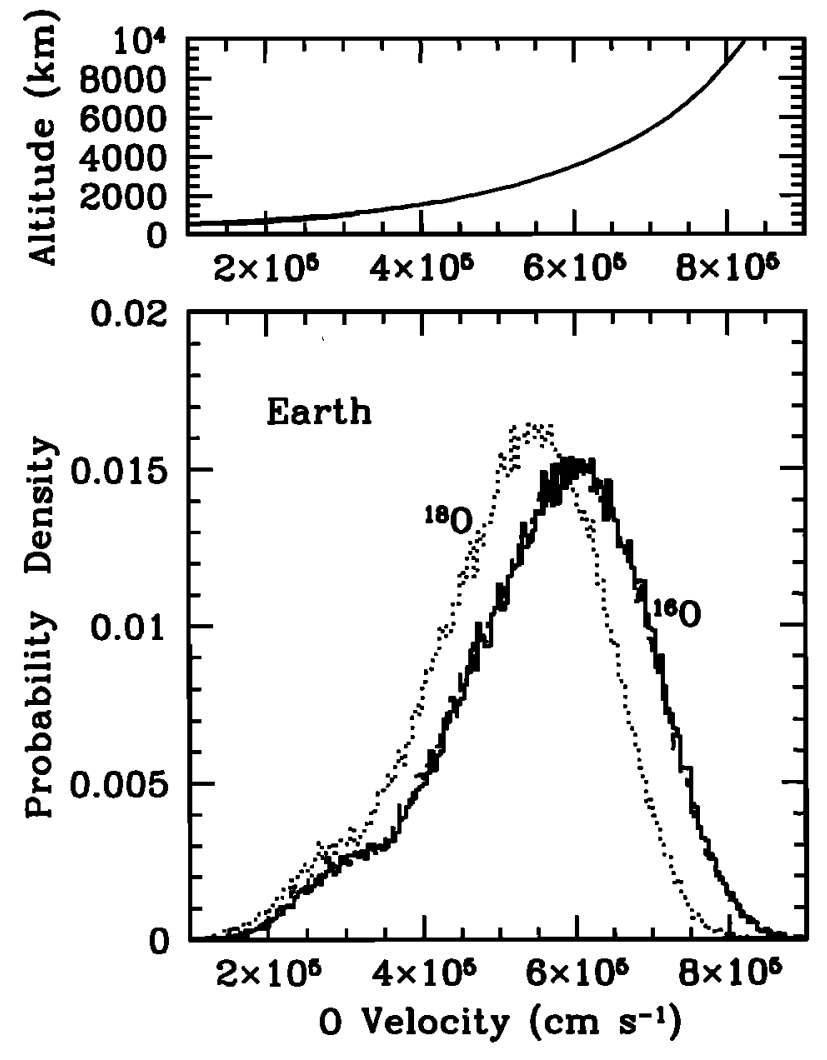

Figure 3. (top) Maximum altitude to which an $O$ atom released vertically at the terrestrial exobase can rise as a function of velocity. (bottom) Nascent velocity distribution of ${ }^{18} \mathrm{O}$ and ${ }^{16} \mathrm{O}$ for the terrestrial low solar activity exobase, near $450 \mathrm{~km}$. The ion and electron temperatures are 1200 and $2700 \mathrm{~K}$, respectively. The rotational temperature $T_{r}$ is assumed to be equal to the ion temperature. The peaks for the individual channels other than channel (1b) are barely visible, due to the higher ion temperature, and the broader vibrational distribution. The dashed curve is the velocity spectrum for $T_{r}=10 \mathrm{~K}$ and can barely be distinguished from the curve for $T_{r}=T_{i}$.

velocity side of the central peak, and those for channels (1d) and (1e) appear as shoulders on the low-velocity side. As for Venus, this is due largely to the higher exobase ion temperature and to the more extended vibrational distribution. Because the escape velocities for Venus and Earth exceed $10 \mathrm{~km} \mathrm{~s}^{-1}$, no escape of either isotope is predicted, but the peaks in the velocity distributions of ${ }^{18} \mathrm{O}$ and ${ }^{16} \mathrm{O}$ differ substantially, and consequently the altitude distributions will be slightly different.

We also show in Figure 3 the velocity distribution of ${ }^{16} \mathrm{O}$ for $T_{r}=10 \mathrm{~K}$, which essentially removes the effect of rotational energy. The velocities are only slightly smaller, averaging $5.581 \mathrm{~km} \mathrm{~s}^{-1}$, compared to that those for $T_{r}=T_{i}$, which are $5.626 \mathrm{~km} \mathrm{~s}^{-1}$ on average. Thus the assumed rotational distribution is not significant, but may be more important for larger rotational temperatures. The major factors in determining the final velocity distribution of the $\mathrm{O}$ atoms are the exother- micity of the reaction and the ion temperature. The exothermicity of the reaction is affected significantly by the vibrational energy level of the ion, but much less by its rotational energy and the kinetic energy of the electron.

Although some models of hot $\mathrm{O}$ coronas have incorporated a broadening of the initial velocities [e.g., Nagy and Cravens, 1988; Hodges et al., 1993], in some of the models it appears that the assumed $O$ velocity is a delta function determined solely by the energy released in the reaction. In many of the models, the branching ratios adopted are quite different from the Kella et al. [1997] measured values. None of the models up to the present have taken the vibrational or rotational distribution of the $\mathrm{O}_{2}^{+}$into account, or the decrease in cross section with increase in relative velocity of the ion and electron.

We have presented here only the velocity spectrum at the exobase. Significant coronal atom production will occur from about one scale height below the exobase to two or more scale heights above, depending upon the altitude profiles of the $\mathrm{O}_{2}^{+}$ions and electrons. The nascent velocity distributions could conceivably differ substantially over this range. We are at present carrying out more detailed calculations of the $O$ velocity distributions for a range of altitudes and for the other sources of hot $\mathrm{O}$, such as $\mathrm{NO}^{+} \mathrm{DR}$, and the photochemical sources suggested by Richards et al. [1994] and Hickey et al. [1995].

Acknowledgments. This work has been supported in part by grant NAGW-2958 to the State University of New York at Stony Brook. The investigation was initiated several years ago under a grant from the National Science Foundation to MIT.

The Editor thanks W. R. Hoegy and another referee for their assistance in evaluating this paper.

\section{References}

Alge, E., N. G. Adams, and D. Smith, Measurements of the dissociative recombination coefficients of $\mathrm{O}_{2}^{+}, \mathrm{NO}^{+}$, and $\mathrm{NH}_{4}^{+}$in the temperature range $200-600 \mathrm{~K}, J$. Phys. B., $16,1433,1983$.

Bilitza, D., and W. R. Hoegy, Solar activity variations of ionospheric plasma temperatures, Adv. Space Res., 10(8), 81-90, 1990.

Bisikalo, D. V., V. I. Shematovich, and J. C. Gérard, A kinetic model of the formation of the hot oxygen geocorona, 2, Influence of $\mathrm{O}^{+}$precipitation J. Geophys. Res., 100, 3715-3720, 1995.

Cotton, D. M., G. R. Gladstone, and S. Chakrabarti, Sounding rocket observation of a hot atomic oxygen geocorona, J. Geophys. Res., 98, 21,651-21,657, 1993.

Fox, J. L., The $\mathrm{O}_{2}^{+}$vibrational distribution in the Venusian ionosphere, Adv. Space Res., 5(9), 165, 1985.

Fox, J. L., The $\mathrm{O}_{2}^{+}$vibrational distribution in the dayside ionosphere, Planet. Space Sci. 34, 1252, 1986.

Fox, J. L., The production of hot oxygen at the exobases of the terrestrial planets, Eos Trans. $A G U, 71(43)$ Fall Meet. Suppl., 1492, 1990.

Fox, J. L., On the escape of oxygen and hydrogen from Mars, Geophys. Res. Lett., 20, 1747, 1993. 
Fox, J. L., and S. W. Bougher, Structure, luminosity and dynamics of the Venus thermosphere, Space Sci. Rev., $55,357-489,1991$.

Fox, J. L., and A. Hać, ${ }^{15} \mathrm{~N} /{ }^{14} \mathrm{~N}$ Isotope fractionation in $\mathrm{N}_{2}^{+}$ dissociative recombination, J. Geophys. Res., 102, 91919204, 1997.

Guberman, S. L., Potential energy curves for dissociative recombination, in Physics of Ion-Ion and Electron-Ion Collisions, edited by F. Brouillard and J. W. McGowan, pp. 167-200, Plenum, New York, 1983.

Guberman, S. L., The production of $O\left({ }^{1} S\right)$ in dissociative recombination of $\mathrm{O}_{2}^{+}$, Nature, 327, 408, 1987.

Guberman, S. L., The production of $O\left({ }^{1} D\right)$ from dissociative recombination of $\mathrm{O}_{2}^{+}$, Planet. Space. Sci. 36, 47, 1988.

Hanson, W. B., and G. P. Mantas, Viking electron temperature measurements: Evidence for a magnetic field in the Martian atmosphere, J. Geophys. Res., 93, 7538, 1988.

Hanson, W. B., S. Sanatani, and D. R. Zuccaro, The Martian ionosphere as observed by the Viking retarding potential analyzers, J. Geophys. Res., 82, 4351, 1977.

Hickey, M. P., P. G. Richards, and D. G. Torr, New Sources for the hot oxygen geocorona: Solar cycle, seasonal, latitudinal and diurnal variations, J. Geophys. Res., 100, 17,377-17,388, 1995.

Hodges, R. R., Isotope fractionation of hydrogen in planetary exospheres due to ionosphere-exosphere coupling: Implications for Venus, J. Geophys. Res., 98, 10,833$10,838,1993$.

Hunton, D. E., A. A. Viggiano, R. A. Morris, and J. F. Paulson, The $\mathrm{O}^{+}+\mathrm{CO}_{2}$ reaction: New results and atmospheric implications, J. Geophys. Res., 96, 13,88113,886, 1991.

Ip, W.-H., On a hot oxygen corona of Mars, Icarus, 76, $135-145,1988$.

Ip, W.-H., The fast atomic oxygen corona extent of Mars, Geophys. Res. Lett., 17, 2289-2292, 1990.

Jakosky, B. M., Mars volatile evolution: Evidence from stable isotopes, Icarus, 94, 14-31, 1992.

Kella, D., P. J. Johnson, H. B. Pedersen, L. Vejby-Christensen and $\mathrm{L}$. H. Andersen, The source of green light emission determined from a heavy-ion storage ring experiment, Science, 276, 1530, 1997.

Laher, R. R., and F. R. Gilmore, Improved fits for the vibrational and rotational constants of many states of nitrogen and oxygen, J. Phys. Chem. Ref. Data, 20, 685-712, 1991.

Lammer, H., and S. J. Bauer, Nonthermal atmospheric escape from Mars and Titan, J. Geophys. Res., 96, 1819, 1991.

McElroy, M. B., Mars: An evolving atmosphere, Science, $175,443,1972$.

McElroy, M. B., and Y. L. Yung, Oxygen isotopes in the Martian atmosphere: Implications for the evolution of volatiles, Planet. Space Sci., 24, 1107, 1976.

McElroy, M. B., M. J. Prather, and J. M Rodriguez, Loss of oxygen from Venus, Geophys. Res. Lett. 9, 649, 1982.

Miller, K. L., W. C. Knudsen, K. Spenner, R. C. Whitten, and V. Novak, Solar zenith angle dependence of the ionospheric ion and electron temperatures and density on Venus, J. Geophys. Res., 85, 7759-7764, 1980.

Mul, P. M., and J. W. McGowan, Temperature dependence of dissociative recombination for atmospheric ions $\mathrm{NO}^{+}$, $\mathrm{O}_{2}^{+}, \mathrm{N}_{2}^{+}, J$. Phys. B, 12, 1591, 1979.

Nagy, A. F., and T. E. Cravens, Hot oxygen atoms in the upper atmosphere of Venus and Mars. Geophys. Res. Lett. 15, 433, 1988.
Nagy, A. F., J. Kim, and T. E. Cravens, Hot hydrogen and oxygen atoms in the upper atmospheres of Venus and Mars. Ann. Geophys. 8, 251, 1990.

Nagy, A. F., T. E. Cravens, J.-H. Yee, and A. I. F. Stewart, Hot oxygen atoms in the upper atmosphere of Venus, Geophys. Res. Lett., 8, 629, 1981.

Nier, A. O., M. B. McElroy, and Y. L. Yung, Isotopic composition of the Martian atmosphere, Science 194, 68, 1976.

Paxton, L. J., Atomic carbon in the Venus thermosphere: Observations and theory, Ph.D. thesis, Univ. of Colo. Boulder, 1983.

Paxton, L. J., and D. E. Anderson, Far ultraviolet remote sensing of Venus and Mars, in Venus and Mars: Atmospheres, Ionospheres, and Solar Wind Interactions, Geophys. Monogr. Ser., vol. 66, edited by J. G. Luhmann et al., pp. 113-189, AGU, Washington, D. C., 1992.

Richards, P. G., M. P. Hickey, and D. G. Torr, New sources for the hot $\mathrm{O}$ geocorona, Geophys. Res. Lett., 21, 657$660,1994$.

Rohrbaugh, R. P., and J. S. Nisbet, Effect of energetic oxygen atoms on neutral density models, J. Geophys. Res., $78,6768,1973$.

Shematovich, V. I., D. V. Bisikalo, and J. C. Gerard, A kinetic model of the formation of the hot oxygen geocorona, 1. Quiet geomagnetic conditions, J. Geophys. Res., 99, 23,217-23,228, 1994.

Wallis, M. K., Exospheric density and escape fluxes of atomic isotopes on Venus and Mars, Planet. Space Sci., 26, 949, 1978.

Wallis, M. K., Comet-like interaction of Venus with the solar wind. III. The atomic oxygen corona, Geophys. Res. Lett., 9, 427, 1982.

Walls, F. L., and G. H. Dunn, Measurement of total cross section for electron recombination with $\mathrm{NO}^{+}$and $\mathrm{O}_{2}^{+}$using ion storage techniques, J. Geophys. Res., 79, 1911, 1974.

Walter, C. W., P. C. Cosby, and J. R. Peterson, Rovibrational product distributions of $\mathrm{O}_{2}^{+}$from the reaction of $\mathrm{O}^{+}\left({ }^{4} S\right)$ with $\mathrm{CO}_{2}, J$. Chem. Phys., 98, 2860, 1993.

Yee, J.-H., and P. B. Hays, The oxygen polar corona, J. Geophys. Res., 85, 1795, 1980.

Yee, J.-H., J. W. Meriwether, and P. B. Hays, Detection of a corona of fast oxygen atoms during solar maximum, $J$. Geophys. Res., 85, 3396, 1980.

Zahnle, K. J., and J. C. G. Walker, The evolution of solar ultraviolet luminosity, Rev. Geophys., 20, 280, 1982.

Zipf, E. C., The dissociative recombination of $\mathrm{O}_{2}^{+}$ions into specifically identified states, Bull. Am. Phys. Soc., 15, 498, 1970.

Zipf, E. C., The excitation of the $\mathrm{O}\left({ }^{1} S\right)$ state by the dissociative recombination of $\mathrm{O}_{2}^{+}$ions: electron temperature dependence, Planet. Space Sci., 36, 621, 1988.

Zhang, M. H. G., J. G. Luhmann, S. W. Bougher, and A. F. Nagy, The ancient oxygen exosphere of Mars: Implications for atmosphere evolution, J. Geophys. Res., 98, $10,915,1993$.

J. L. Fox, Department of Physics, Wright State University, Dayton, OH 45435. (email: fox@platmo.phy.wright.edu)

A. Hać, Delphi Chassis Technical Center, Mail Code E520, 1435 Cincinnati St., Dayton, OH 45401.

(Received April 18, 1997; revised July 17, 1997; accepted July 18, 1997.) 